\title{
A simple phenotypic classification for celiac disease
}

\author{
Ajit Sood ${ }^{1}$, Vandana Midha ${ }^{2}$, Govind Makharia ${ }^{3}$, B. K. Thelma ${ }^{4}$, Shivalingappa S Halli ${ }^{5}$, Varun Mehta ${ }^{1}$, \\ Ramit Mahajan $^{1}$, Vikram Narang ${ }^{6}$, Kriti Sood $^{6}$, Kirandeep Kaur $^{7}$ \\ Departments of ${ }^{1}$ Gastroenterology and ${ }^{2}$ Internal Medicine, Dayanand Medical College, Ludhiana, ${ }^{3}$ Department of Gastroenterology and \\ Human Nutrition, All India Institute of Medical Sciences, New Delhi, ${ }^{4}$ Department of Genetics, University of Delhi South Campus, New \\ Delhi, India, ${ }^{5}$ Department of Community Health Sciences, Faculty of Medicine, University of Manitoba, Winnipeg, Canada, Departments of \\ ${ }^{6}$ Pathology and ${ }^{7}$ Pharmacology, Dayanand Medical College, Ludhiana, India
}

\begin{abstract}
Background/Aims: Celiac disease is a global health problem. The presentation of celiac disease has unfolded over years and it is now known that it can manifest at different ages, has varied presentations, and is prone to develop complications, if not managed properly. Although the Oslo definitions provide consensus on the various terminologies used in literature, there is no phenotypic classification providing a composite diagnosis for the disease. Methods: Various variables identified for phenotypic classification included age at diagnosis, age at onset of symptoms, clinical presentation, family history and complications. These were applied to the existing registry of 1,664 patients at Dayanand Medical College and Hospital, Ludhiana, India. In addition, age was evaluated as below 15 and below 18 years. Cross tabulations were used for the verification of the classification using the existing data. Expert opinion was sought from both international and national experts of varying fields. Results: After empirical verification, age at diagnosis was considered appropriate in between A1 $(<18)$ and A2 ( $\geq 18)$. The disease presentation has been classified into 3 types-P1 (classical), P2 (non-classical) and P3 (asymptomatic). Complications were considered as absent (C0) or present (C1). A single phenotypic classification based on these 3 characteristics, namely age at the diagnosis, clinical presentation, and intestinal complications (APC classification) was derived. Conclusions: APC classification (age at diagnosis, presentation, complications) is a simple disease explanatory classification for patients with celiac disease aimed at providing a composite diagnosis. (Intest Res 2018;16:288-292)
\end{abstract}

Key Words: Celiac disease; Classification; Presentation; Complications

\section{INTRODUCTION}

Celiac disease is an immune-mediated enteropathy characterized by intolerance to gluten and similar proteins of barley and rye in genetically susceptible individuals. In the past, celiac disease was considered a rare disorder affecting persons mainly of European origin. ${ }^{1,2}$ However, in the last decade and a half, the disease has moved from obscurity to a popular spotlight, being one of the most common treatable autoimmune disorders to affect humans. The disease has heterogeneous clinical presentations. ${ }^{3,4}$ The classic constel-

Received August 30, 2017. Revised January 1, 2018

Accepted January 3, 2018. Published online January 31, 2018

Correspondence to Ajit Sood, Department of Gastroenterology, Dayanand

Medical College \& Hospital, Tagore Nagar, Ludhiana 141001, India. Tel: +91

9815400718,Fax:+91-161-2302620; E-mail: ajitsood10@gmail.com lation of symptoms and signs which characterize malabsorption, i.e., chronic diarrhea, abdominal bloating, failure to thrive and so forth are the so-called typical, classical, diarrheal or intestinal forms of disease presentation. ${ }^{5}$ Other diverse ways in which celiac disease manifests such as fatigue, iron deficiency anemia, short stature, osteomalacia or osteoporosis, cryptogenic liver disease etc. are variously described as atypical, extra intestinal, non-diarrheal or non-classic presentations of celiac disease. ${ }^{6}$ Other terminologies used are silent group, "at-risk," "potential celiac disease" and "latent celiac disease." ${ }^{7}$ Similarly the age of onset/diagnosis labels a case of celiac disease as pediatric celiac disease or adult celiac disease with varied cutoffs as distinction between the two. This variation in terminologies used to define gluten related disorders invoked interest of international experts who got together to provide simple definition for celiac disease

(c) Copyright 2018. Korean Association for the Study of Intestinal Diseases. All rights reserved.

This is an Open Access article distributed under the terms of the Creative Commons Attribution Non-Commercial License (http://creativecommons.org/licenses/by-nc/4.0)

which permits unrestricted non-commercial use, distribution, and reproduction in any medium, provided the original work is properly cited. 
and related terms in their recent "Oslo definitions." ${ }^{\prime 8}$ The use of terms typical, atypical, silent and latent celiac disease was discouraged. The focus of Oslo group was to clarify the use of terminologies in relation to gluten related disorders and not disease characterization or classification in relation to various parameters.

In clinical practice, just labeling a case of celiac disease according to presentation alone or according to age of diagnosis alone does not give a lucid description of the disease. Hence, we believe that more stringent adoption of consistent, standardized classification of celiac disease according to relevant variables is necessary in the modern era of data collection and disease management. Moreover, a classification system would provide a common language with which professionals can discuss patients, regardless of their geographical location. Celiac disease, though known for decades, still presents a challenge for classifying individuals as no definite classifiers have been recognized and put together in a meaningful fashion. Till date, no phenotypic classification has been used for celiac disease patients. Therefore, an attempt is made in this paper to develop a clinical phenotypic classification system for celiac disease.

\section{METHODS}

For the purpose of a simple phenotypic classification aimed at providing a composite diagnosis of celiac disease, our team considered different parameters for disease characterization. Various attributes of the patients that our group agreed for classifying disease phenotype included age at diagnosis, age at onset of symptoms, disease presentation, and complications. The study was approved by the Institutional Review Board (IRB No. 2014-78 DMC/R\&D-2015/220), informed consent was not needed.

\section{Definitions}

Age at diagnosis was defined as the age at establishment of diagnosis of celiac disease based on modified ESPGAN criteria. ${ }^{9}$ Age at the time of onset of symptoms was also recorded. The disease presentation was sub-classified as (1) P1 (classical celiac disease)-patients who presented with diarrhea, failure to thrive and features of malnutrition and/ or malabsorption syndrome; (2) P2 (non-classical celiac disease)-patients who lacked features included in P1 (classical form) and presented with either or combination of medical conditions like unexplained iron deficiency anemia, osteoporosis, short stature, infertility, cryptogenic liver disease, dermatitis herpetiformis and neurological symptoms, and so forth; (3) P3 (latent celiac disease)-patients with positive celiac disease serology but with normal duodenal histology; and (d) P4 (screen detected)-patients who were detected during screening of "at-risk" population. Patients with both intestinal and extraintestinal manifestations were included in the first group (P1, classical celiac disease). The disease complications were divided as either $\mathrm{C} 0$-no complications or C1-with complications like refractory sprue, ulcerative jejunoileitis, celiac crisis or malignancy.

\section{Data Analysis}

The cutoffs for age for defining pediatric cases (i.e., $<18$ or $<15$ years) were analyzed separately. In addition, cross tabulations were used for the verification of both the age at onset of the symptoms and the age at diagnosis of celiac disease. The variables for the proposed classification were applied to our data registry which included 1,664 patients with confirmed diagnosis of celiac disease.

In addition, it was decided to be sent to 30 recognized national and international experts on celiac disease for opinion. The experts included 22 gastroenterologists, 3 pediatricians, 4 physicians and 1 professor of genetics (working on genetics of celiac disease in children and adults). There were areas of consensus and areas where there was need for consideration before a final consensus.

\section{RESULTS}

Our dataset of 1,664 patients were classified according to age groups and clinical presentation. First tabulation consisted of 2 variables: the age of diagnosis with 2 categories such as $<18$ years and $\geq 18$ years by the reference classification variable, disease presentation. Similarly, the second included age groups as $<15$ years and $\geq 15$ years along with the same reference classification variable. As can be seen in

Table 1. Classification of Celiac Disease Patients by Age Groups and Disease Presentation

\begin{tabular}{rrrrrr}
\hline \multirow{2}{*}{$\begin{array}{c}\text { Age } \\
\text { (yr) }\end{array}$} & \multicolumn{4}{c}{ Presentation } & \multirow{2}{*}{ Total } \\
\cline { 2 - 5 } & P1 & \multicolumn{1}{c}{ P2 } & P3 & P4 & \\
\hline$<15$ & $369(81.09)$ & $79(17.36)$ & $4(0.87)$ & $3(0.65)$ & $455(100)$ \\
$\geq 15$ & $1,038(85.85)$ & $152(12.57)$ & $9(0.74)$ & $10(0.82)$ & $1,209(100)$ \\
$<18$ & $447(81.12)$ & $95(17.24)$ & $4(0.72)$ & $5(0.90)$ & $551(100)$ \\
$\geq 18$ & $960(86.25)$ & $136(12.21)$ & $9(0.80)$ & $8(0.71)$ & $1,113(100)$ \\
\hline
\end{tabular}

Values are presented as number (\%). 
Table 1 below, the row percentages for the corresponding age categories are almost identical implying that it does not make any difference whether the cutoff criteria is $\leq 15$ years or $\leq 18$ for characterization of celiac disease into pediatric and adult onset.

Again, the 2 variables, i.e., age at onset of symptoms and age at diagnosis were analyzed (Table 2). Surprisingly, we found that $93 \%([547+1,005] / 1,664)$ of the 1,664 cases were the same between the 2 variables for a given grouping of the age variable suggesting that there is a good concordance between age of onset of symptoms and age of diagnosis.

Hence, it was agreed to use the following classifiers: age at diagnosis of disease, presentation and complications. Of the 20 experts who responded to the e-mail 12 were gastroenterologists, 4 physicians and 3 pediatricians and 1 professor of genetics. Substantive comments from experts included the basis for 18 years as cutoff. In order to incorporate their concern, we have explained at length the rationale for setting 18 years as the cutoff age in the discussion section. The other concerns regarding presentation sub-categories included P3 to be deleted, P1 and P2 overlap and nomenclature of $\mathrm{P} 1$ and P2 as diarrheal and non-diarrheal. In addition, one expert had concern about the inclusion of complications in the classification as it needed investigative work-up and oc-

Table 2. Classification of Celiac Disease Patients by Age at Diagnosis and Age of Onset of Symptoms

\begin{tabular}{cccc}
\hline \multirow{2}{*}{ Age at diagnosis } & \multicolumn{2}{c}{ Age at onset of symptoms } & \multirow{2}{*}{ Total } \\
\cline { 2 - 3 } & $\mathbf{< 1 8} \mathbf{~ y r}$ & $\mathbf{2 1 8} \mathbf{~ y r}$ & \\
\hline$<18 \mathrm{yr}$ & 547 & 4 & 551 \\
$\geq 18 \mathrm{yr}$ & 108 & 1,005 & 1,113 \\
Total & 655 & 1,009 & 1,664 \\
\hline
\end{tabular}

curred in later stages. Majority of the experts provided strong support for the classification. They also indicated in their return reply along with their tabular responses that the classification is simple and elegant and can be very useful for both practitioners and researchers alike.

After taking into consideration the suggestions made by the experts, the classification was modified as shown in Table 3. It was agreed to delete P3 as latent disease and P4 as silent disease or screen detected and in turn, to rename P3 as asymptomatic disease. Using these variables on the database for classification we could identify our patients as A1P1C0 447, A1P2CO 95, A1P3C0 9, A2P1C0 955, A2P1C1 5, A2P2C0 131, A2P2C1 5, and A2P3C0 17. Intestinal complications were identified in 10 patients above the age of 18 , were jejunoileitis $(n=2)$, celiac crisis $(n=2)$, refractory sprue $(n=1)$, carcinoma esophagus $(n=1)$, stomach $(n=1)$, ovary $(\mathrm{n}=1)$, small bowel $(\mathrm{n}=1)$, and nodal lymphoma $(\mathrm{n}=1)$.

\section{DISCUSSION}

Various factors known to influence the course of celiac disease and its long-term prognosis are: age at diagnosis of disease, disease presentation and complications. The age of diagnosis may reflect the severity of disease and perhaps, associated complications. Disease management and prognosis change vastly once these complications set in. Furthermore, disease presentation can be an easy guide for individualized management and follow-up plan for each patient. For instance, for a patient presenting with osteoporosis, the treating physician would like to follow-up with serial DEXA (dual energy X-ray absorptiometry) scans while serial hemogram monitoring would be required for a patient who had iron deficiency anemia as the presenting feature. Disease presentation also matters as asymptomatic patients may not be very

Table 3. APC Classification for Celiac Disease

\begin{tabular}{ll}
\hline Age at diagnosis & A1: $<18$ yr of age \\
& A2: $\geq 18$ yr of age \\
Presentation & P1: Classical. Chronic diarrhea, failure to thrive, malnutrition or malabsorption syndrome \\
& P2: Non-classical. Lack of features included in classical disease. Presentation with extraintestinal medical conditions \\
& like unexplained iron deficiency anemia, osteoporosis, short stature, infertility, cryptogenic liver disease, dermatitis \\
& herpetiformis, neurological disorders etc \\
& P3: Asymptomatic \\
Complications & C0: None \\
& C1: Present. Complications like refractory sprue, ulcerative jejunoileitis, celiac crisis or malignancy \\
\hline
\end{tabular}

$\mathrm{APC}$, age at diagnosis, presentation and complications. 
much willing to accept major dietary modifications resulting in poor dietary compliance. With such a wide range of disease presentations having impact on disease behavior, it becomes imperative that disease pattern of each patient needs to be categorized individually. Hence, it is of paramount importance to characterize each patient's disease according to these factors since just writing a diagnosis of celiac disease does not provide required information about the disease. In order to achieve this and ensure a composite diagnosis we are suggesting a phenotypic classification based primarily on 3 variables, i.e., age at diagnosis, presentation and complications (APC classification) (Table 3). For instance, a 30-yearold patient presenting as iron deficiency anemia diagnosed as celiac disease will be classified as A2P2 (iron deficiency anemia) $\mathrm{C} 0$. The classification remains open to change in category on serial follow-ups. Similarly, development of intestinal complications would result change in category from C0 to $\mathrm{Cl}$.

Based on extensive review of literature and our own clinical experience, age was considered as one of the important parameters. With respect to the "cutoff age" for defining pediatric cases, we argue that it would be appropriate to take children who are less than 18 years of age. However, there is no consensus on this as some researchers argue in favor of age $\leq 15$ years. Moreover, we were guided by the "Convention on the Rights of the Child," adopted by the United Nations General Assembly on 20 November, 1989. According to article 1 of the convention, "a child means every human being below the age of 18 years unless, under the law applicable to the child, majority is attained earlier." ${ }^{\prime 10}$ Furthermore, according to Indian Association of Pediatric Policy Statement on Age of Children for Pediatric Care (1999): "For fulfilling the professional obligations of pediatricians to the society at large, the purview of pediatrics commences with the fetus and continues through newborn, infancy, preschool and school age including adolescents up to and including 18 years of age." ${ }^{11}$

Another challenge was to choose between the 2 factors, i.e., the age at onset of the disease and the age at diagnosis for the classification exercise. Although it was desirable to choose the age at onset of the symptoms for age characterization but it would have entailed a recall bias, more so among older patients and hence we considered age at diagnosis for classification. Moreover, this corroborates with findings of other researchers. ${ }^{12}$

We think that this classification provides clinically relevant information with regard to the disease so as to have a composite diagnosis. Overall, useful applications of this classification can be (1) phenotypic characterization of disease for each patient; (2) individualized disease management and follow-up for each patient; (3) more precise monitoring of the disease behavior; (4) highlighting of the disease related complications resulting in a close follow-up of such patients with specific investigations; (5) better identification of disease pattern in different populations or countries with a uniformity in disease classification; (6) easier recognition of changing trends in disease presentation; and (7) characterization of more homogenous subgroups for research purposes.

We have attempted to formulate a disease explanatory simple classification for patients with celiac disease. This classification scheme is not related to disease prognosis and makes no reference to histology or genetics. It is anticipated that as we learn more about the disease and put this classification into practice, further validation by other centers will be useful. All classifications tend to have some inconsistencies or inaccuracies and we believe that the present effort is no exception.

\section{FINANCIAL SUPPORT}

The authors received no financial support for the research, authorship, and/or publication of this article.

\section{CONFLICT OF INTEREST}

No potential conflict of interest relevant to this article was reported.

\section{AUTHOR CONTRIBUTION}

Conceptualization: A.S., Vandana Midha. Methodology: A.S., Vandana Midha, G.M. Writing-original draft: AS, Vandana Midha. Writing-review and editing: all authors.

All authors approved the final version of the manuscript.

\section{ACKNOWLEDGEMENTS}

The authors would like to thank Dr S Bhatia, Dr Ganesh Pai, Dr V. K. Dixit, Dr V. Jayanthi, Dr. P Mathur, Dr. Nijhawan, Dr. S. P. Malhotra, Dr. Vineet Ahuja, Dr. K. L. Goh, Dr. Cisca, Dr. Carlo, Dr. Mulder, Dr. Sandeep Puri, Dr. Dinesh Gupta, Dr. H. S. Pannu, Dr. Narender Pal Jain, Dr. B. R. Thapa, Dr. Yaccha, Dr. Puneet Pooni and Dr. BK Thelma for their valuable opinions on the feasibility, applicability, practicability and clinical usability of the classification. 


\section{REFERENCES}

1. Losowsky MS. A history of coeliac disease. Dig Dis 2008;26:112120.

2. Green PH, Cellier C. Celiac disease. N Engl J Med 2007;357: 1731-1743.

3. Rewers M. Epidemiology of celiac disease: what are the prevalence, incidence, and progression of celiac disease? Gastroenterology 2005;128(4 Suppl 1):S47-S51.

4. Oberhuber G, Granditsch G, Vogelsang H. The histopathology of coeliac disease: time for a standardized report scheme for pathologists. Eur J Gastroenterol Hepatol 1999;11:1185-1194.

5. Fasano A. Clinical presentation of celiac disease in the pediatric population. Gastroenterology 2005;128(4 Suppl 1):S68-S73.

6. Fasano A. Celiac disease: how to handle a clinical chameleon. N Engl J Med 2003;348:2568-2570.
7. Ferguson A, Arranz E, O'Mahony S. Clinical and pathological spectrum of coeliac disease-active, silent, latent, potential. Gut 1993;34:150-151.

8. Ludvigsson JF, Leffler DA, Bai JC, et al. The Oslo definitions for coeliac disease and related terms. Gut 2013;62:43-52.

9. Revised criteria for diagnosis of coeliac disease: report of Working Group of European Society of Paediatric Gastroenterology and Nutrition. Arch Dis Child 1990;65:909-911.

10. United Nations General Assembly. Convention on the Rights of the Child, 20 November 1989. Annu Rev Popul Law 1989;16:95, 485-501.

11. John TJ. IAP policy on age of children for pediatric care. Indian Pediatr 1999;36:461-463.

12. Gasche C, Scholmerich J, Brynskov J, et al. A simple classification of Crohn's disease: report of the Working Party for the World Congresses of Gastroenterology, Vienna 1998. Inflamm Bowel Dis 2000;6:8-15. 